\title{
El Nuevo Proceso Constitucional de la Acción de Tutela en Modalidad Virtual para la Protección del Derecho Fundamental a la Salud en Tiempos del COVID - $19^{1}$
}

\author{
The New Constitutional Process of the Protection \\ Action in Virtual Modality for the Fundamental Right to \\ Health Protection in Times of COVID - 19
}

\section{Carlos Alfredo Pérez Fuentes}

Doctorando en Educación y Estudios Sociales

Institución Universitaria Tecnológico de Antioquia abog.carlosperez@gmail.com

\footnotetext{
1 El presente artículo de investigación inédito es producto del proyecto de investigación de tesis doctoral titulado "Análisis de la fundamentación filosófica de los diseños curriculares de programas de derecho en Colombia", el cual fue realizado en el marco del Semillero de Investigación en Estudios Jurídicos Interdisciplinarios del Tecnológico de Antioquia. Esta investigación fue presentada en ponencia en el V Congreso Nacional e Internacional de Semilleros Grupos de Investigación y Redes de la Asociación de Facultades de Derecho de Colombia.
} 


\title{
Annie Julieth Álvarez Maestre
}

\author{
Doctora en Educación \\ Institución Universitaria Tecnológico de Antioquia \\ anniejulieth@gmail.com
}

\section{Andrea Johana Aguilar-Barreto}

Doctora en Educación

Institución Universitaria Tecnológico de Antioquia

andreitajaguilar@hotmail.com

\section{Daniel Felipe Rodríguez Montoya}

Estudiante de Derecho

Institución Universitaria Tecnológico de Antioquia

rodriguez.felipe5050@gmail.com

\footnotetext{
Cómo citar este artículo:

Pérez, C. A., Álvarez, A. J., Aguilar, A. J., y Rodríguez, D. F. (2022). El Nuevo Proceso Constitucional de la Acción de Tutela en Modalidad Virtual para la Protección del Derecho Fundamental a la Salud en Tiempos del COVID - 19. Revista de la Facultad de Derecho y Ciencias Políticas, 52(136), pp. 219-238. doi: https://doi.org/10.18566/rfdcp.v52n136.a09
}

Recibido: 08 de octubre 2020

Aprobado: 27 de mayo de 2021 


\section{Resumen}

El presente artículo de investigación es producto de la investigación titulada "Eficacia del derecho fundamental a la salud en el contexto del proceso constitucional virtual en tiempo del COVID-19 en Colombia", la cual tiene primer objetivo reconocer el nuevo proceso constitucional de la acción de tutela bajo la modalidad virtual para la protección del derecho fundamental a la salud. De esta forma, en este artículo se presentarán los avances de esta investigación los cuales pretenden reconocer las características propias de este nuevo proceso constitucional para la protección del derecho fundamental a la salud, creado por los Acuerdos expedidos por el Consejo Superior de la Judicatura.

Esta investigación se desarrolló bajo un enfoque cualitativo y un método hermenéutico que pretendió comprender holísticamente el significado de los escritos relacionados con la temática conservando la singularidad de estos, a través de matrices de análisis que permiten descomponer los textos para comprenderlos como un todo integrado.

Como resultados parciales se puede observar que el nuevo "proceso constitucional de tutela virtual" representa la flexibilización en un contexto de ductilidad en donde la adaptación mediada por las TIC ha permitido la garantía de los derechos fundamentales a través de la no suspensión de términos en materia de tutelas, presentación de la acción por correo electrónico, prevalencia del derecho fundamental a la vida, la salud y la libertad, entre otras.

\section{Palabras clave}

Derecho fundamental, derecho fundamental a la salud, COVID-19, acción de tutela, eficacia.

\section{Abstract}

This research article is the product of the research study called "The fundamental right to health effectiveness in the virtual constitutional process context in times of COVID-19 in Colombia", whose first objective is to recognize the new constitutional process of the protection action under the virtual modality for the fundamental right to health protection.

Thus, this article will describe the advances of this research which seeks to recognize the characteristics of this new constitutional process for the fundamental right to health protection, created by the Agreements issued by the Superior Council of the Judicature.

This research was developed under a qualitative approach and a hermeneutical method that sought to comprehensively understand the meaning of the writings related to the subject while preserving their uniqueness, through analysis matrixes that allow to decompose the texts to understand them. 
As partial results, it can be observed that the new "constitutional process of virtual protection action" represents flexibility in a context of ductility where adaptation mediated by ICTs has allowed the assurance of fundamental rights through the non-suspension of terms of protection actions, to file the action by email, prevalence of the fundamental right to life, health, and liberty, among others.

\section{Keywords}

Fundamental right; fundamental right to health; COVID-19; protection action, efficacy.

\section{Introducción}

La Cuarta Revolución Industrial ha traído consigo enormes cambios derivados del uso de las Tecnologías de la Información y la Comunicación (en adelante, TIC), por lo que estas tecnologías han ayudado enormemente a mitigar los impactos del COVID-19 a nivel mundial.

En el campo del Derecho, las TIC han llegado para aportar a la reducción de los problemas de congestión judicial generados a partir de la acumulación y demoras procesales, esto, mediante la realización de juicios digitales, actuaciones procesales virtuales, entre otros., que pretende garantizar en la mayor medida de los posible los principios de transparencia, celeridad y economía procesal.

De esta forma, ante la contingencia sanitaria del COVID-19 la administración de justicia en Colombia ha tenido que expedir una serie de Acuerdos que crearon nuevas reglas en los procesos judiciales. El caso que ocupa a este artículo es el de la creación de nuevas reglas procesales que dieron origen a un proceso constitucional de tutela virtual, el cual será objeto de estudio en las páginas siguientes.

\section{Fundamentación metodológica}

La presente investigación se desarrolla bajo un enfoque cualitativo debido a que: "trata de identificar, básicamente, la naturaleza profunda de las realidades, su estructura dinámica, aquella que da razón plena de su comportamiento y manifestaciones" (Martínez, 2004, p. 66,). A su vez, se desarrolla bajo un método hermenéutico que pretende 
[...] descubrir los significados de las cosas, interpretar lo mejor posible las palabras, los escritos, los gestos y, en general, el comportamiento humano, así como cualquier otro acto u obra suya, pero conservando su singularidad en el contexto de que forma parte. (Martínez, 2004, p. 102).

Para la recolección de la información se tuvo en cuenta la técnica de revisión documental que se concibe como: "un proceso que abarca la ubicación, recopilación, selección, revisión, análisis, extracción y registro de información contenida en documentos” (Hurtado, 2010, p. 851). Finalmente, el análisis de dicha información se realizará a través de la técnica de análisis del discurso que pretende descomponer en sus partes la información recolectada para comprenderla como un todo.

\section{Planteamiento del problema}

El COVID-19 ha traído consigo enormes cambios en los que el ser humano debe adaptarse aceleradamente para continuar con su forma de vida. Para ello, las Tecnologías de la Información y de la Comunicación (en adelante, TIC) han resultado ser herramientas confiables que permiten minimizar el impacto de la pandemia en los diferentes escenarios de la vida.

En este contexto, la Cuarta Revolución Industrial surge en un punto en el que a través de la interdisciplinariedad se pretende optimizar los procesos humanos a través de la innovación y las TIC, ya que la virtualidad permite mantener la cotidianidad de los procesos a través de las redes sociales, plataformas virtuales de trabajo y demás, el cambio abrupto derivado de la pandemia ha traído consigo diversos traumas en los procesos judiciales, entre estos: problemas de adaptabilidad (Schwab, 2016); ralentización de procesos debido a la falta de conocimiento técnico (Ríos, 2017); exclusión por causas económicas, sociales o culturales (Schwab, 2016), arbitrariedades y abusos en el procesos judiciales en la virtualidad (Navarrete, 2017), nacimiento y vulneración de derechos fundamentales en el contexto de la virtualidad (Bachelet, 2019; Antúnez, 2019), necesidad de implementar avances tecnológicos en la administración de justicia (Sanjurjo, 2011).

Ante estas problemáticas surge la pregunta de investigación: ¿Cuál es la perspectiva de eficacia de los jueces constitucionales frente al proceso constitucional de tutela virtual para la garantía del derecho fundamental a la salud? 


\section{Fundamentación Teórica}

\section{Cuarta Revolución Industrial en el mundo.}

Debido a las circunstancias que se presentan actualmente a raíz de la propagación del virus COVID-19 a nivel mundial, el ser humano ha tenido que vivir un proceso de adaptación acelerado a las nuevas herramientas y escenarios que se presentan en el mundo virtual con el fin de mitigar el impacto que de la pandemia surge en todas las esferas de la sociedad.

Así, la cuarta revolución industrial se presenta como el escenario donde confluyen las fuerzas físicas, digitales y biológicas que buscan optimizar de manera interdisciplinar el rendimiento de los procesos humanos por medio de la innovación y la tecnología (Schwab,2016), lo cual permite a los individuos, en tiempos de la pandemia, mantener la cotidianidad de sus comportamientos a través de la virtualidad por medio de las redes sociales, plataformas virtuales de trabajo, redes económicas de distribución y consumo, entre otros. (Gasca y Machuca, 2019; Ríos 2017).

Teniendo en cuenta la evolución desmesurada de las herramientas tecnológicas pertenecientes a la Cuarta Revolución Industrial en general y con mayor rapidez a raíz de la propagación del COVID-19, gran parte de la población a nivel mundial se ha visto afectada de manera negativa por no tener acceso a las herramientas necesarias para obtener beneficios de la era digital, lo que representa a su vez la posibilidad que tienen las grandes industrias e instituciones del Estado para usar sus recursos en pro de intereses particulares; escenario que muestra el necesario desarrollo del Derecho como elemento evolutivo que debe intervenir para regular las arbitrariedades y los abusos que pueden resultar de los avances tecnológicos. (Navarrete, 2017). Todos los operadores jurídicos juegan un papel fundamental en el proceso adaptativo y de creación y aplicación de reglas que permitan un desarrollo equitativo de la era digital. (Navarrete, 2017).

En este contexto, se busca actualizar el proceso judicial a fin que los individuos tengan un mejor y eficaz acceso a la Justicia a través de las herramientas virtuales que propone la nueva era digital, puesto que, si bien es complejo lograr la sincronía entre los avances tecnológicos y económicos con su regulación a través de los sistemas jurídicos, se hace necesario buscar al menos ciertos objetivos que disminuyan los alcances exacerbados del mundo virtual; fines como los juicios en línea, la optimización del capital 
humano para atender las demandas, la reducción de los materiales físicos por material virtual legal, acceso público a las actuaciones judiciales, hacer control ciudadano de forma virtual en los procesos de la administración judicial y pública, entre otros, permitirán dinamizar el ordenamiento jurídico y los procesos buscando ductilidad, transparencia, celeridad y efectividad real. (Ríos, 2017; Arteaga, 2018; Priori, 2015).

\section{Los Derechos Fundamentales y su Relación con los Derechos Sociales Fundamentales}

Con el desarrollo del Estado Social de Derecho se da una modificación en los ordenamientos jurídicos que lo adoptan con la aparición del estatus supremo de la Constitución, por encima de la ley, como norma fundamental de cada Estado. En ésta se desarrolla un catálogo de Derechos Fundamentales que, en suma, son aquellos derechos inherentes a la persona que tienen como objetivo primordial asegurar la dignidad del individuo son inviolables y deben ser garantizados por el Estado y los demás participes de la sociedad (Bernal, 2015; Alexy, 1993). Sin embargo, debido a la complejidad respecto de su aplicación en casos concretos se debe anotar que no son materia acabada, ya que presentan problemas de fundamentación racional, vaguedad conceptual y complementación de las demás disposiciones del derecho positivamente válido. (Alexy, 1993, p. 30).

En este punto, se reconocen derechos fundamentales como la libertad y la dignidad en donde se reclama una no-intervención por parte del Estado en las actuaciones de los individuos y, los derechos sociales fundamentales los cuales se reconocen como aquellos que representan una facultad que tiene el individuo de reclamar del Estado (Alexy, 1993) actuaciones o prestaciones positivas, toda vez que, no tiene las herramientas o recursos para satisfacer de manera propia necesidades básicas que representan en conjunto la posibilidad del individuo de subsistir. (Arango, 2015).

Así pues, se han determinado dentro de la categoría de Derechos Sociales Fundamentales el derecho a la educación, el trabajo, la salud, la vivienda, la seguridad social, entre otros (Arango, 2015; Arango, s/f), como aquellos que, si bien, en algunas Constituciones no se consagran al nivel de los demás Derechos Fundamentales como la libertad y la autonomía, aun así, tienen rango fundamental en el desarrollo y el logro de los planes de vida que son de elección individual. (Nino, 2007). 
En tiempos del COVID-19 se decretó en Colombia el Estado de Emergencia Sanitaria, escenario que ha modificado el desarrollo de la sociedad en múltiples niveles. Ejemplo de ello, son las actividades desarrolladas en el marco de los Derechos Sociales Fundamentales, en los que se evidencia una afectación compleja en términos de educación, acceso al trabajo, salud y demás. (Pérez Fuentes y Álvarez Maestre, 2020)

Adicional a ello se debe anotar que, en los procesos judiciales de reclamación de estos derechos, también se ha presentado una afectación que no permite el desarrollo normal de los trámites, ya que se vulneran de manera amplia estas prerrogativas que representan un deber de hacer para el Estado como "Agencia Colectiva por excelencia para satisfacer las prestaciones positivas fácticas objeto de los Derechos Sociales Fundamentales”. (Arango, 2015, p. 1688).

En este contexto, la justiciabilidad de los derechos debe comprenderse históricamente a partir del rango iusfundamental de los Derechos Sociales Fundamentales, el cual se ha logrado a partir de luchas sociales y políticas (Arango, s/f) que han alejado la concepción de Derechos que se deben reconocer por vía de la conexidad con un Derecho Fundamental (Arango, s/f) por no estar determinados como Derechos Fundamentales de la Ley Fundamental (Alexy, 1993).

Hoy en día, se han desarrollado por vía de la Jurisprudencia Constitucional tanto el reconocimiento del estatus fundamental de los Derechos Sociales como la Salud, a través de mecanismos como la acción de tutela (Arango, s/f, p.17) para proteger su justiciabilidad, por medio de los cuales, los titulares del Derecho pueden hacer efectiva su protección en los sistemas de administración de justicia del Estado. (Pérez, 2020).

\section{Estructuración del trabajo}

Para llevar a cabo la presente investigación se planteó como objetivo general de la investigación: Comprender la perspectiva del Juez Constitucional frente a la eficacia del proceso constitucional de tutela bajo la modalidad virtualidad en torno a la protección del derecho fundamental a la salud.

Para desarrollar este objetivo, se plantearon tres objetivos específicos, a saber: a) reconocer el proceso constitucional de la acción de tutela bajo 
la modalidad virtual con énfasis en la protección del derecho fundamental a la salud desarrollado por el Consejo Superior de la Judicatura en tiempos del COVID-19; b) verificar el cumplimiento de las ordenes emitidas por los jueces constitucionales a través del incidente de desacato; c) reconocer las perspectivas del juez constitucional frente a la eficacia del derecho fundamental a la salud en el proceso constitucional de tutela virtual.

\section{El Proceso Constitucional de Tutela Bajo la Modalidad Virtual con Énfasis en la Protección del Derecho Fundamental a la Salud Desarrollado por el Consejo Superior de la Judicatura en Tiempos del Covid-19.}

Generalidades del Proceso Constitucional de la Acción de Tutela.

Conforme al Decreto 2591 de 1991 se reglamenta el artículo 86 de la Constitución Política de 1991 sobre la acción de tutela. En este contexto, esta acción pretende que toda persona pueda reclamar ante los jueces, en todo momento y lugar, la protección inmediata de sus derechos constitucionales fundamentales a través de un procedimiento preferente y sumario, cuando considere que estos están siendo vulnerados por cualquier autoridad o particular. (art. 1).

De este modo, en el procedimiento de la acción de tutela se pueden distinguir cuatro etapas, i) la procedencia de la acción de tutela, entendida como aquellos presupuestos necesarios para la presentación de la acción de tutela, ii) la presentación de la solicitud, la cual se refiere a los trámites necesarios para la presentación, correcciones y notificaciones a las partes en el proceso, iii) el fallo, que consiste en la decisión del juez restablecer inmediatamente un derecho, proteger un derecho tutelado, prevenir a la autoridad y generar la indemnización y costas y, finalmente, iv) cumplimiento de fallo, el cual cumple la función de verificar la ejecución de las órdenes del Juez Constitucional, es decir, que el accionado cumpla dentro de los términos establecidos por la Ley y la Constitución.

Conforme a lo anteriormente, mencionado se esboza la Figura 1, en la cual se resalta el papel del fallo y el cumplimiento del fallo, ya que allí, se concentra el concepto de eficacia de la sentencia del Juez Constitucional. 
Figura 1. Procedimiento de la acción de tutela.

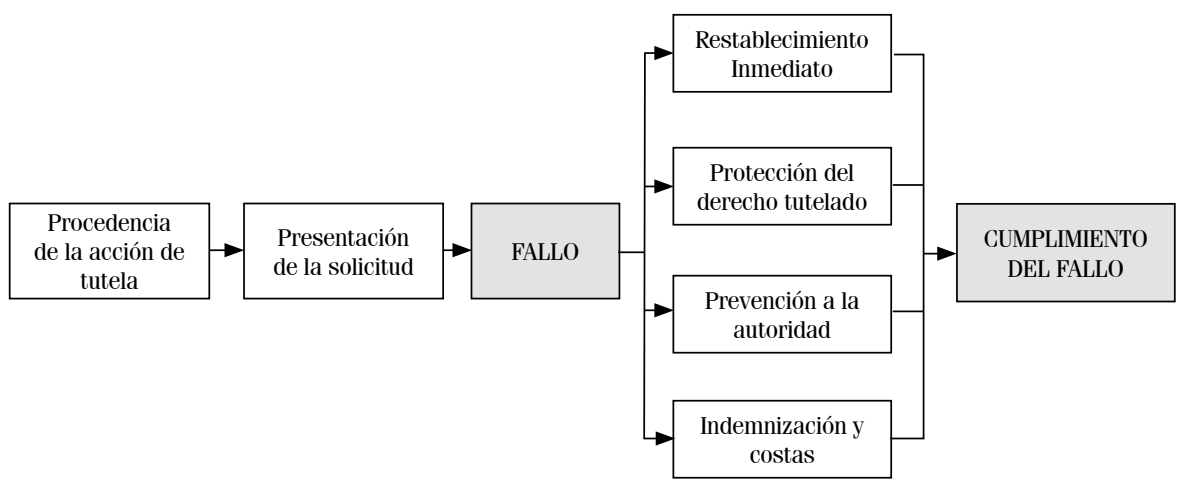

Fuente: Elaborador por los autores.

En este sentido, la eficacia de la protección de los derechos fundamentales se concentra en el cumplimiento del fallo, razón por la cual, los jueces constitucionales reconocen y establecen el incumplimiento al hacer uso de las medidas establecidas en el artículo 27 del Decreto 2591 de 1991², sancionando el incumplimiento de las sentencias de tutela a través de lo establecido en esta normatividad.

\section{El Proceso Constitucional de Tutela Modalidad Virtual en el Sistema Jurídico Colombiano.}

Esta normatividad relacionada con el proceso constitucional de tutela nace a partir de la declaración de urgencia manifiesta del Acuerdo PCSJA20-11516 del 12 de marzo de 2020 del Consejo Superior de la Judicatura. Al respecto, menciona el Tribunal que, esta medida se genera debido a emergencia sanitaria por el COVID-19 a nivel mundial, razón

2 Esta norma establece: articulo 27. Cumplimiento del fallo. Proferido el fallo que conceda la tutela, la autoridad responsable del agravio deberá cumplirla sin demora. Si no lo hiciere dentro de las cuarenta y ocho horas siguientes, el juez se dirigirá al superior del responsable y le requerirá para que lo haga cumplir y abra el correspondiente procedimiento disciplinario contra aquél. Pasadas otras cuarenta y ocho horas, ordenará abrir proceso contra el superior que no hubiere procedido conforme a lo ordenado y adoptará directamente todas las medidas para el cabal cumplimiento del mismo. El juez podrá sancionar por desacato al responsable y al superior hasta que cumplan su sentencia. Lo anterior sin perjuicio de la responsabilidad del funcionario en su caso. En todo caso, el juez establecerá los demás efectos del fallo para el caso concreto y mantendrá la competencia hasta que esté completamente restablecido el derecho o eliminadas las causas de la amenaza. [énfasis agregado] 
por la cual la Corporación se encontraría en la necesidad de implementar medidas responsables que ayuden a controlar y prevenir la situación de salud pública a nivel nacional.

Posteriormente, el 12 de marzo de 2020 el Consejo emite Acuerdo PCSJA20-11517 a través del cual: se suspenden los términos judiciales en todo el país desde 16 al 20 de marzo, excepto en los juzgados penales que deberán realizar las audiencias virtualmente y, el trámite de las acciones de tutela. Al respecto, en dicho Acuerdo se establece que los magistrados, jueces y jefes de dependencias administrativas coordinarán y darán las instrucciones para los servidores labores desde sus casas.

Luego, el 16 de marzo de 2020 se expide el Acuerdo PCSJA20-11518 3 y el Acuerdo PCSJA20-11519, el cual suspende los términos de la revisión eventual de tutelas desde el 17 al 20 de marzo, por lo que los despachos no remitirán los expedientes de acciones de tutela a la Corte Constitucional hasta que no se levanten las medidas adoptadas.

El 19 de marzo de 2020 a través del Acuerdo PCSJA20-115214 se prorroga las medidas de los Acuerdos anteriormente mencionados, desde el 21 de marzo al 03 de abril y se incluyen las excepciones dispuestas. El 22 de marzo de 2020 el Acuerdo PCSJA20-11526 ${ }^{5}$ prorroga nuevamente la suspensión de términos desde el 04 de abril hasta el 12 de abril, en este Acuerdo se resaltan las acciones de tutela y habeas corpus, las cuales son una excepción a dicha suspensión:

3 A través del Acuerdo PCSJA20-11518 del día 16 de marzo de 2020 el Consejo Superior mantiene las medidas de suspensión anteriormente mencionadas y, exceptúa las acciones de tutela y habeas corpus. En este Acuerdo, en su artículo 2 se establece que los funcionarios continuarán trabajando desde sus casas y a su vez, se llevará a cabo la activación de correos electrónicos por despacho judicial; el juez, magistrado o jefe dirigirá al equipo de trabajo en la asignación de labores y designará a un empleado que éste atento para la recepción de documentos, éste no podrá ser mayor de 60 años, mujer gestante o lactante o alguna enfermedad que genere situaciones de vulnerabilidad según la Organización Mundial de la Salud.

4 Los magistrados, jueces y empleados judiciales seguirán laboran desde sus casas y excepcionalmente, podrán acudir a las sedes judicial para adelantar actividades específicas. Los cuerpos colegiados de las Altas Cortes y Tribunales del país podrán hacer reuniones de trabajo y sesiones de trabajo, estableciendo reglas propias para su desarrollo.

5 Además de lo anterior, el Consejo Superior refiere que los Consejos Seccionales de la Judicatura y direcciones seccionales de la administración de judiciales seguirán prestando el apoyo técnico a los servidores judiciales y administrativos para que puedan adelantar sus actividades con el apoyo de herramientas y medios tecnológicos, de preferencia institucionales. (Art. 4) El Consejo Superior publicaría los lineamientos básicos para el uso de las TIC y el reparto de las tutelas se suspendería a los juzgados penales municipales con función de control de garantías y de ejecución de penas y medidas de seguridad en el país. 
Acciones de tutela y habeas corpus:

Se dará prelación en el reparto a las acciones de tutela que versen sobre derechos fundamentales a la vida, la salud y la libertad. Su recepción se hará mediante correo electrónico dispuesto para el efecto y para su trámite y comunicaciones se hará uso de las cuentas de correo electrónico y herramientas tecnológicas de apoyo. (Consejo Superior de la Judicatura, Acuerdo PCSJA20-11526, art. 2) [énfasis agregado].

A través del Acuerdo PCSJA20-11527 del 22 de marzo de 2020, se exceptúan de la suspensión las actuaciones adelantadas por la Corte Constitucional relacionadas con la expedición de Decretos por parte del presidente de la República. El mismo día, mediante Acuerdo PCSJA20$11528^{6}$ se suspenden los términos de las actuaciones administrativas a desarrollar por la Dirección Ejecutiva de Administración y direcciones seccionales. El 25 de marzo de 2020, el Acuerdo PCSJA20-11529 se exceptúa a la suspensión de términos del control inmediato de legalidad del Consejo de Estado y Tribunales Administrativos de las competencias establecidas en el artículo 20 de la Ley 137 de 1994 y en los artículos 111, numeral 8; 136 y 151, numeral 14, del Código de Procedimiento Administrativo y de lo Contencioso Administrativo.

Posteriormente, a través del Acuerdo PCSJA20-11532 ${ }^{7}$ del 11 de abril de 2020 se prorrogó la suspensión de términos desde el 13 de abril hasta el 26 de abril de 2020. En este Acuerdo se mantienen la suspensión de la excepción de la acción de tutela (art. 2), la prestación del servicio desde casa (art. 5) y el uso de las TIC (art. 6). En estos casos, se establecen las siguientes reglas especiales:

1. La recepción, gestión, trámite, decisión y actuaciones judiciales y administrativas se privilegiará el uso de las TIC;

2. Los jueces preferencialmente utilizarán las TIC para todas las actuaciones procesales;

6 Entre las actuaciones administrativas se encuentran: procesos administrativos de cobro coactivo, procesos disciplinarios, reclamaciones de depósitos judiciales, procesos administrativos salariales y prestacionales.

$7 \quad$ Este Acuerdo fue emitido comprendiendo que el presidente de la República a través del Decreto 417 de 2020 declaró "Estado de Emergencia Económica, Social y Ecológica" en todo territorio nacional, el Decreto Legislativo 491 del 28 de marzo de 2020 que buscó adoptar medidas de urgencia para garantizar la prestación de servicios por parte de las autoridades y el Decreto Legislativo 531 del 08 de abril de 2020 que ordenó el aislamiento preventivo obligatorio desde el 13 de abril hasta el 27 abril. 
3. Los memoriales podrán ser enviados y recibidos por correo electrónicos, para evitar presentaciones personales. En la mayor medida de lo posible, en formato PDF;

4. Las partes, abogados, terceros e intervinientes en los procesos judiciales y administrativos deberán suministrar su correo electrónico para recibir comunicaciones y notificaciones;

5. Conforme al Decreto 491 de 2020, las firmas podrán ser digitales, digitalizadas o escaneadas y todos los órganos de las ramas del poder público podrán realizar reuniones no presenciales para deliberar y decidir un asunto.

Además de ello, en este Acuerdo se dispone que el Consejo Superior de la Judicatura a través del Centro de Documentación Judicial asegure por lo menos una cuenta de correo electrónico institucional para cada despacho judicial, secretarias, oficinas de apoyo, centro de servicios y demás dependencias. Los despachos deberán publicar los estados por la página web de la Rama Judicial, por lo que se deberán adecuar estos espacios. Finalmente, se mantiene la exoneración de reparto de tutelas y habeas corpus a los juzgados penales municipales y, además, cuando sea necesario asignar durante los fines de semana estos mecanismos se realizará entre los despachos que se encuentran en turno o disponibles, agregando a los juzgados anteriormente mencionados.

El Acuerdo PCSJA20-11546 ${ }^{8}$ del 25 de abril de 2020 prorroga la suspensión de términos desde el 27 de abril hasta el 10 de mayo de 2020. El Acuerdo PCSJA20-11548 del 05 de abril de 2020 que adopta la creación de algunos cargos para algunos juzgados y centros de servicios de ejecución de penas y medidas de seguridad nivel nacional. El Acuerdo PCSJA20-11556 del 22 de mayo de 2020, el cual prorroga la suspensión de términos judiciales desde el 25 de mayo hasta el 08 de junio de 2020 , ampliando las excepciones establecidas en el Acuerdo PCSJA20-11546 del 25 de abril de 2020.

8 Además, este Acuerdo amplía las excepciones establecidas en acciones de tutela y habeas corpus, control de constitucionales de decretos legislativos, en lo contencioso administrativo, penal, civil, familia, laboral, disciplinario, Dirección Ejecutiva de Administración Judicial. Por lo demás, mantiene el trabajo preferente en casa y el uso de las TIC en todas las actuaciones procesales. 
El Acuerdo PCSJA20-11567 ${ }^{9}$ del 05 junio de 2020 a través del cual se levanta la suspensión de términos judiciales y administrativos en todo el país a partir del 01 de julio de 2020, por lo que los servidores podrán acudir a las sedes con el fin de finalizar tareas de planeación y organización del trabajo bajo las condiciones de este acuerdo. En este sentido, se prorrogan la suspensión desde 09 de junio hasta el 30 de junio, exceptuando las acciones relacionadas anteriormente y ampliando aún más procesos en los que se suspenden los términos. En este Acuerdo se establecen reglas generales para el acceso y permanencia en las sedes, condiciones de bioseguridad, condiciones para el trabajo virtual y condiciones de trabajo en casa.

i. Es importante mencionar que este Acuerdo establece:

ii. El uso preferente de las TIC,

Los aplicativos de recepción de tutelas, habeas corpus y firmas electrónicas se utilizarán mientras dure la suspensión de términos, el envío de tutelas será por correo electrónico;

iii. Se privilegiarán las audiencias virtuales,

iv Los órganos podrán llevar a cabo reuniones no presenciales,

v.. Los depósitos judiciales se coordinarán con el Banco Agrario,

vi. La atención al usuario se realizará por medios electrónicos como correo electrónico o llamadas telefónicas u otros

Finalmente, se encuentran los Acuerdos PCSJA20-11571 del 19 de junio de 2020 a través del cual se crean ciertos cargos en la Dirección Ejecutiva de Administración Judicial relacionados con Ingeniería de Sistemas para construir soluciones tecnológicas mediante el desarrollo de software que pueda recibir todo tipo de demandas judiciales y sea interactivo con las partes y la ciudadanía. Y, el Acuerdo PCSJA20-11581 del 27 de junio de 2020 a través del cual se dictan disposiciones especiales sobre el levantamiento de términos, en este se establece que:

\footnotetext{
9 Es importante mencionar que, en este Acuerdo preferentemente el trabajo es en casa, aunque se puede dar la presencialidad de máximo el $20 \%$ de servidores judiciales por despacho en las sedes, esto será organizado por el Magistrado, Juez o jefe según las necesidades del despacho, aquellos que padezcan ciertas enfermedades definidas por el Decreto no podrán asistir en ninguna circunstancia a las sedes. A partir del 17 de junio se establecerán horarios de atención al público dependiendo las características de la ciudad o región.
} 
i. Se mantiene la suspensión de términos del Acuerdo PCSJA20-11567

ii. Las sedes judiciales y administrativas de la Rama Judicial no prestarán atención presencial al público, para ello los Consejos Seccionales darán a conocer los medios y canales de comunicación para los trámites, si se requiere ingresar solo se hará en los horarios establecidos y con el respeto a los protocolos de bioseguridad

iii. La correspondencia física será recibida por las direcciones seccionales de administración de justicia

Conforme a lo anteriormente, mencionado se genera la Figura 2, la cual pretende describir la evolución histórica de las decisiones tomadas a través de Acuerdos por parte del Consejo Superior de la Judicatura frente a la contingencia por el COVID-19.

Ahora bien, sobre los Acuerdos se pueden concluir que:

1. Una vez se emite el Acuerdo PCSJA20-11516 que declaró la "urgencia manifiesta”, el mismo día el Consejo Superior de la Judicatura emitió el Acuerdo PCSJA20-11517 a través de cual se suspendieron todos los términos judiciales con excepciones a los juzgados penales y procesos de tutela

2. A través de los Acuerdos PCSJA20-11518 y Acuerdo PCSJA20-11521 se prorrogan y mantienen excepciones. Además, se dan instrucciones sobre el trabajo en casa, el Magistrado, Juez o jefe deberá dirigir al equipo de trabajo en asignación de labores y los cuerpos colegiados podrán realizar sesiones no presenciales

3. En el Acuerdo PCSJA20-11526 se generan reglas especiales para las acciones de tutela y habeas corpus. Se da la prelación a acciones de tutela relacionadas con el derecho a la vida, la salud y libertad. Su recepción y trámite se realizará por correo electrónico, y con el apoyo de las demás herramientas tecnológicas

4. A través de los Acuerdos PCSJA20-11527, PCSJA20-11528 y PCSJA20-11529 se exceptúan actuaciones de la Corte Constitucional, Dirección Ejecutiva de la Administración de Justicia, Consejo de Estado y Tribunales Administrativos

5. A través del Acuerdo PCSJA20-11532 se mantienen las excepciones y se establecen reglas especiales, tales como: el privilegio en el uso de las TIC, el uso del correo electrónico, los memoriales en lo posible en PDF, los correos electrónicos de las partes, las firmas y las reuniones no presenciales para la toma de decisiones. Los despachos tendrán al menos un correo institucional, los estados se publicarán por la página web de 


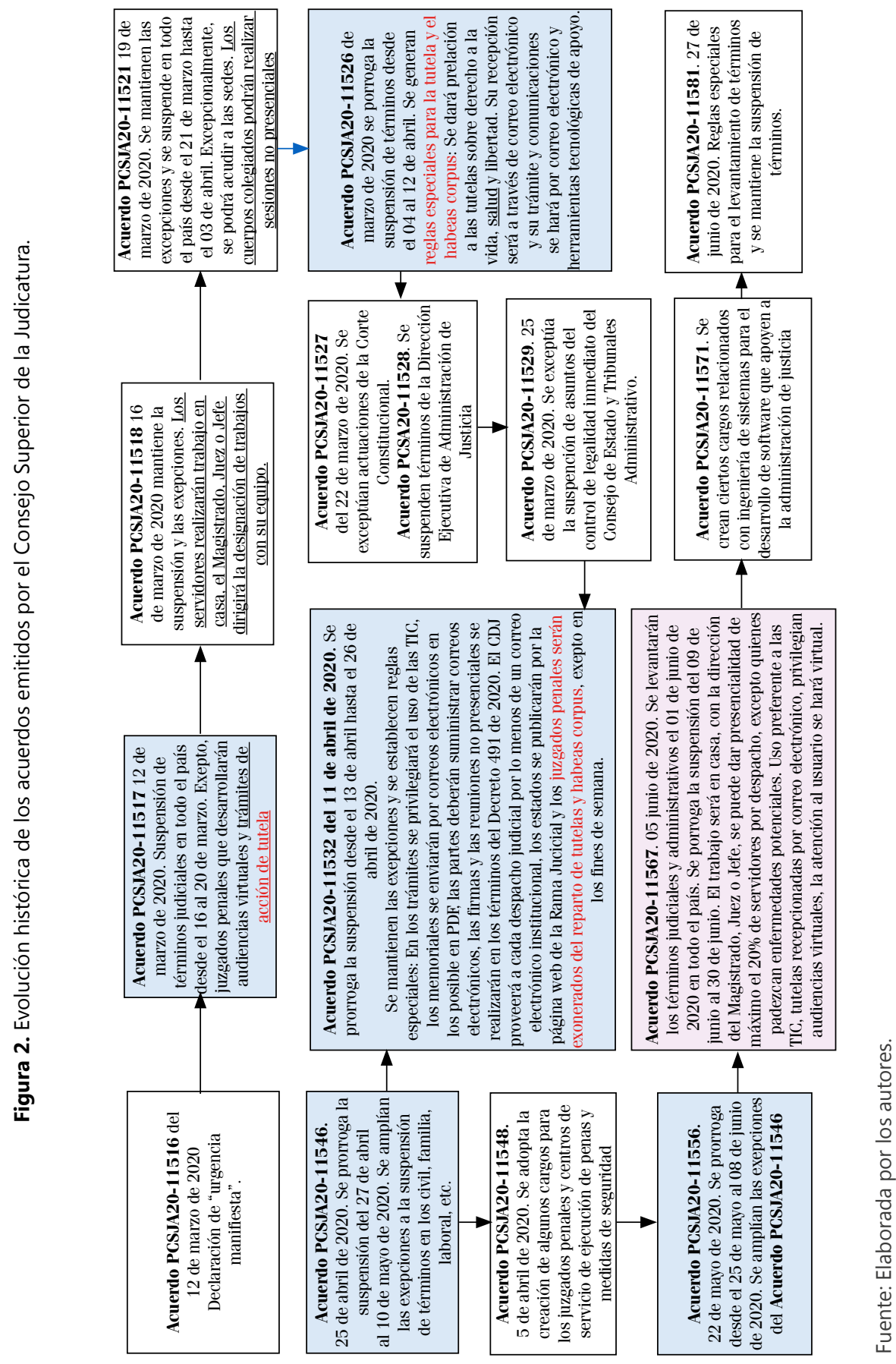


la Rama Judicial y los juzgados penales serán exonerados del reparto de acciones de tutela y habeas corpus

6. Los Acuerdos PCSJA20-11546, PCSJA20-11548 y PCSJA20-11556 amplían los términos para otras actuaciones procesales y suspenden términos

7. El Acuerdo PCSJA20-11567 suspendió términos hasta el 01 de junio, ya que este día se levantarían todos los términos. Las tutelas se recibirán por correo electrónico y se privilegiará el uso de las TIC, audiencias virtuales, atención virtual al usuario, excepcionalmente atención presencial en sedes

8. Los Acuerdos PCSJA20-11571 y PCSJA20-11581 refieren creación decargos para el mejoramiento de los programas software de la Administración de Justicia y reglas especiales para el levantamiento de términos.

\section{Conclusión}

En conclusión, frente al proceso constitucional de tutela del Decreto 2591 de 1991 se observan ciertas adiciones que los autores de este trabajo denominan el nuevo "proceso constitucional de tutela virtual", el cual representa la flexibilización del proceso en un contexto de ductilidad ${ }^{10}$ en donde la adaptación mediada por las TIC ha permitido la garantía de los derechos fundamentales en Colombia. Este proceso virtual aplicado en época de cuarentena tiene las siguientes características:

1. La presentación de la acción de tutela puede realizarse a través del correo electrónico del despacho judicial, el cual se encuentra publicado en la página de la Rama Judicial

2. Los términos procesales de la acción de tutela nunca se suspendieron

3. Las acciones de tutela sobre los derechos fundamentales a la vida, la salud y la libertad son preferentes frente a las demás

10 Para desarrollar el concepto de "proceso dúctil" es necesario mencionar que se toma la perspectiva desarrollada a partir del artículo científico titulado "El proceso dúctil" del Dr. Giovanni F. Priori Posada publicado en el libro del XXXVI Congreso Colombiano de Derecho Procesal realizado en Pereira (Colombia) en el año 2015, ISSN: 2322 - 6560. Para el autor, el proceso dúctil se concibe como una concepción del proceso judicial en donde los derechos preceden al proceso, ya que en éste "la tutela jurisdiccional no es una forma abstracta, indiferente a las características de la situación sustancial necesitada de tutela, sino que, por el contrario, es un aspecto necesariamente concreto que se va modelando en torno a las particularidades y exigencias de protección de la situación jurídica material objeto del proceso". (p. 994) Es por esto, que se pretende la maximización de los derechos fundamentales procesales a través de la flexibilización de las reglas procesales, adecuándolas a la protección de los principios constitucionales y los derechos fundamentales demandados por las partes en el proceso; de aquí, que el rol del juez sea conseguir la real eficacia de los derechos fundamentales con decisiones basadas en juicios argumentativos adecuados a las características del proceso. 
4. Todo el proceso debe realizarse preferencialmente de forma virtual, ya que en los despachos judiciales solo puede asistir el 20\% del equipo de trabajo, cumpliendo con las normas de bioseguridad, a excepción de quienes padecen enfermedades potencialmente vulnerables ante el COVID-19

5. En este nuevo proceso es flexible e innovador debido a que da privilegio al uso de las TIC, las firmas pueden ser digitales, escaneadas, etc., las partes deben tener correo electrónico, los memoriales deben presentarse en la medida de los posible en PDF, los despachos deben tener al menos un correo institucional

6. Todos los despachos judiciales, a excepción de algunos juzgados penales municipales, recibirán tutelas

7. El fallo y la verificación de su cumplimiento se sigue realizando bajo lo establecido en el Decreto 2591 de 1991

\section{Referencias}

Alexy, R. (1993). Teoría de los Derechos Fundamentales. Centro de estudios constitucionales. Madrid, España. Recuperado de http://www.bibliotecad.info/ wp-content/uploads/2018/08/TEORIA-DE-LOS-DERECHOS-FUNDAMENTALESROBERT-ALEXY.pdf

Antúnez, A. (2019). La industria 4.0. Análisis y estudio desde el Derecho en la 4ta Revolución Industrial. Revista Advocatus, 16(32), 103-131. doi: https://doi. org/10.18041/0124-0102/a.32.5526

Arango, R. (2015). Derechos Sociales, Capítulo 47 en Zamora, F., Rodríguez, J. y Blanco, V. (Ed.). Enciclopedia de Filosofía y Teoría del Derecho (Vol. 2). Recuperado de https://archivos.juridicas.unam.mx/www/bjv/libros/8/3796/27.pdf

Arango, R. (s/f). Justiciabilidad de los Derechos Sociales Fundamentales en Colombia. Aporte a la construcción de un ius constitutionale commune en Latinoamérica. Instituto de Investigaciones Jurídicas UNAM. Recuperado de https://archivos. juridicas.unam.mx/www/bjv/libros/7/3063/4.pdf

Arteaga, F. (2018). La cuarta revolución industrial (4RI): un enfoque de seguridad nacional. Real Instituto Elcano. Recuperado de http://www.realinstitutoelcano. org/wps/wcm/connect/d692309d-d6e2-42d6-9308-fccd5f3feae0/DT122018-Arteaga-Cuarta-revolucion-industrial-enfoque-seguridad-nacional. pdf?MOD=AJPERES\&CACHEID =d692309d-d6e2-42d6-9308-fccd5f3feae0

Bachelet, M. (2019). Derechos humanos en la era digital. ¿Pueden marcar la diferencia? Japan Society. Recuperado de https://www.ohchr.org/SP/NewsEvents/Pages/ DisplayNews.aspx?NewsID $=25158 \&$ LangID $=\mathrm{S}$

Bernal, C. (2015). Derechos Fundamentales, Capítulo 44 en Zamora, F., Rodríguez, J. y Blanco, V. (Ed.). Enciclopedia de Filosofía y Teoría del Derecho (Vol. 2). Recuperado de: https://archivos.juridicas.unam.mx/www/bjv/libros/8/3796/24.pdf 
Carlos Alfredo Pérez F., Annie Julieth Álvarez M., Andrea Johana Aguilar-Barreto, Daniel Felipe Rodríguez M.

Consejo Superior de la Judicatura. (2020). Acuerdo No. PCSJA20-11516 de 2020. Bogotá, Colombia. Recuperado de https://www.ramajudicial.gov.co/web/medidascovid19/acuerdos

Consejo Superior de la Judicatura. (2020). Acuerdo No. PCSJA20-11518 de 2020. Bogotá, Colombia. Recuperado de https://www.ramajudicial.gov.co/web/medidascovid19/acuerdos

Consejo Superior de la Judicatura. (2020). Acuerdo No. PCSJA20-11519 de 2020. Bogotá, Colombia. Recuperado de https://www.ramajudicial.gov.co/web/medidascovid19/acuerdos

Consejo Superior de la Judicatura. (2020). Acuerdo No. PCSJA20-11521 de 2020. Bogotá, Colombia. Recuperado de https://www.ramajudicial.gov.co/web/medidascovid19/acuerdos

Consejo Superior de la Judicatura. (2020). Acuerdo No. PCSJA20-11526 de 2020. Bogotá, Colombia. Recuperado de https://www.ramajudicial.gov.co/web/medidascovid19/acuerdos

Consejo Superior de la Judicatura. (2020). Acuerdo No. PCSJA20-11527 de 2020. Bogotá, Colombia. Recuperado de https://www.ramajudicial.gov.co/web/medidascovid19/acuerdos

Consejo Superior de la Judicatura. (2020). Acuerdo No. PCSJA20-11528 de 2020. Bogotá, Colombia. Recuperado de https://www.ramajudicial.gov.co/web/medidascovid19/acuerdos

Consejo Superior de la Judicatura. (2020). Acuerdo No. PCSJA20-11529 de 2020. Bogotá, Colombia. Recuperado de https://www.ramajudicial.gov.co/web/medidascovid19/acuerdos

Consejo Superior de la Judicatura. (2020) Acuerdo No. PCSJA20-11532 de 2020. Bogotá, Colombia. Recuperado de https://www.ramajudicial.gov.co/web/medidascovid19/acuerdos

Consejo Superior de la Judicatura. (2020). Acuerdo No. PCSJA20-11546 de 2020. Bogotá, Colombia. Recuperado de https://www.ramajudicial.gov.co/web/medidascovid19/acuerdos

Consejo Superior de la Judicatura. (2020). Acuerdo No. PCSJA20-11548 de 2020. Bogotá, Colombia. Recuperado de https://www.ramajudicial.gov.co/web/medidascovid19/acuerdos

Consejo Superior de la Judicatura. (2020). Acuerdo No. PCSJA20-11549 de 2020. Bogotá, Colombia. Recuperado de https://www.ramajudicial.gov.co/web/medidascovid19/acuerdos

Consejo Superior de la Judicatura. (2020). Acuerdo No. PCSJA20-11556 de 2020. Bogotá, Colombia. Recuperado de https://www.ramajudicial.gov.co/web/medidascovid19/acuerdos

Consejo Superior de la Judicatura. (2020). Acuerdo No. PCSJA20-11567 de 2020. Bogotá, Colombia. Recuperado de https://www.ramajudicial.gov.co/web/medidascovid19/acuerdos

Consejo Superior de la Judicatura. (2020). Acuerdo No. PCSJA20-11571 de 2020. Bogotá, Colombia. Recuperado de https://www.ramajudicial.gov.co/web/medidascovid19/acuerdos

Consejo Superior de la Judicatura. (2020). Acuerdo No. PCSJA20-11581 de 2020. Bogotá, Colombia. Recuperado de https://www.ramajudicial.gov.co/web/medidascovid19/acuerdos 
Corte Constitucional. (2008). Sentencia T-760 de 2008. Recuperado de https://www. corteconstitucional.gov.co/relatoria/2008/t-760-08.htm.

Gasca, G. P. y Machuca, L. (2019). Era de la Cuarta Revolución Industrial. RISTI, 34, 11-15. DOI 10.17013/risti.34.0

Navarrete, J. C. (2017). Efectos de la Cuarta Revolución Industrial en el Derecho. Revista Facultad de Jurisprudencia, Pontificia Universidad Católica del Ecuador, 2. Recuperado de: revistarfjpuce.edu.ec/index.php/rfj/article/view/19

Pérez Fuentes, CA y Álvarez Maestre, AJ (2020). Nuevos enfoques de enseñanza basados en el aprendizaje aplicados en la ciencia jurídica, en Albornoz-Arias, N., BermúdezPirela, V., Mojica Acevedo, E. C., Espinel Rubio, G. A., Ramirez Paris Colmenares, X., Rojas Suárez, J. P., \& Agudelo Ibáñez, S. J. Análisis de coyunturas fronterizas en y desde Colombia.

Pérez Fuentes, C. A., Hernández Peñaloza, F. A., Leal Castañeda, K., \& Castillo Calderón, D. F. (2020). Análisis jurisprudencial del derecho a la salud en Colombia. Revista Academia \& Derecho, 10(19), 87-12. Recuperado de: https://doi.org/10.18041/22158944/academia.19.6010

Priori, G. (2015). El Proceso Dúctil en Universidad Libre de Colombia (Ed.), XXXVI Congreso Colombiano de Derecho Procesal. Instituto Colombiano de Derecho Procesal. Bogotá D.C., Colombia

Ríos, A. de los A. (2017) Una visión latinoamericana de la justicia en la era digital \& los medios electrónicos. Revista Electrónica Amicus Curiae, 1(9), 6-21. Recuperado de: http://www.revistas.unam.mx/index.php/amicus/article/view/62483

Sanjurjo, B. (2011). Modernización digital de la Administración de Justicia: especial referencia a la jurisdicción contable. Revista Española de Control Externo, 13 (37), 61-77. Recuperado de: https://dialnet.unirioja.es/servlet/articulo?codigo=3710719

Schwab, K. (2016) La Cuarta Revolución Industrial. El Tiempo Casa Editoral, S.A. Bogotá, Colombia. 